\title{
Damage Evolution Model of Fractured Rock Masses under Freeze-Thaw and Load Condition
}

\author{
Xinghong WU ${ }^{1, a}$ and * Yani $L^{2, b}$ \\ ${ }^{1}$ Construction Management Office, Hubei Engineering University, \\ Xiaogan, Hubei 432000, China \\ 2 School of Civil Engineering, Hubei Engineering University, \\ Xiaogan, Hubei 432000, China \\ asiyu-1979@126.com, blyn2016@hbeu.edu.cn
}

\begin{abstract}
Keywords: Rock, Freeze-thaw experiments, Damage evolution, Uniaxial compression test, Coupling effect.

Abstract. Based on the freeze-thaw(F-T) and uniaxial compression test, the article focuses on the damage evolution model of rock masses under coupling action of F-T and load. In this study, a prediction model of rock under uniaxial compressive tests and F-T action was proposed in accordance with elastic-plastic theory and fatigue damage mechanics. This model was validated by the previous experiments including four kinds of rock samples. The results show that the damage evolution model is more reliable for all the chosen rocks with different geometric parameters of fissures, These conclusions lay the theoretical foundation for further development of damage evolution model of fractured rock under F-T cycles for natural building materials and engineering construction in cold regions.
\end{abstract}

\section{Introduction}

Freeze-thaw action is considered as the main weathering processes of rock masses in cold regions, which causes micro-damage, frost heaving damage, instability and failure of rock masses, and does great harm to the mechanical properties of rock $^{[1]}$. A growing number of geotechnical engineering problems under freezing and thawing condition are to be solved as the rise of the engineering construction in western cold regions. Therefore, it is necessary to study damage evolution mechanisms of rock mass of cracked rock under coupling action of freeze - thaw and load, and this is definitely of great importance for us to conduct engineering projects in cold region.

Many researchers have studied the damage evolution mechanisms of rock mass ${ }^{[2-6]}$. Using the decay constant $(\lambda)$ and half-life $\left(\mathrm{N}_{1 / 2}\right)$ parameters, Mutlutürk ${ }^{[2]}$ proposed a decay model that fitted the test results well; Yavuz et al ${ }^{[3]}$ established a model equation for estimating the index properties of deteriorated carbonate rocks due to freeze-thaw cycles. This model explains decrease in index property of a deteriorated rock depending on its initial engineering property and porosity of rock with the coefficients for a specific index property. Tan et $\mathrm{al}^{[4]}$ investigated the degradation in the mechanical properties of granite as a function of freeze-thaw cycles by uniaxial and triaxial compression tests. Yang ${ }^{[5]}$ Gengshe derived the damage evolution equations of freeze - thaw and loaded rocks, and then analyzed microscopic structure damage; Mu Jianqiao ${ }^{[6]}$ analyzed freeze thaw damage failure mechanism of the quanlity of the rock by macro analyzing method.

The above achievement has raised the mechanical property and damage mechanism and damage evolution model of intact rock, but few papers have been published about the fractured rock which is universal in engineering. Given all this, in this article, mechanical property and damage mechanism of fractured rock are discussed in cold region, Totally four groups of specimens were prepared for F-T cycles and uniaxial compression tests respectively, all the four groups were water-saturated and then respectively suffered 0, 20, 40,60 artificial F-T cycles. Decay models of mechanical properties reflecting were built, and the model parameters were identified based on experimental results. In the last part, some significant conclusions have been drawn from this study. 


\section{Material and Methods}

Sandstone was made into standard cylinder specimens, $\Phi 100 \times 50 \mathrm{~mm}$, and the samples were produced fractured rock with different dip angles, Figure 1 shows the geometric parameters of the single fractured rock samples.
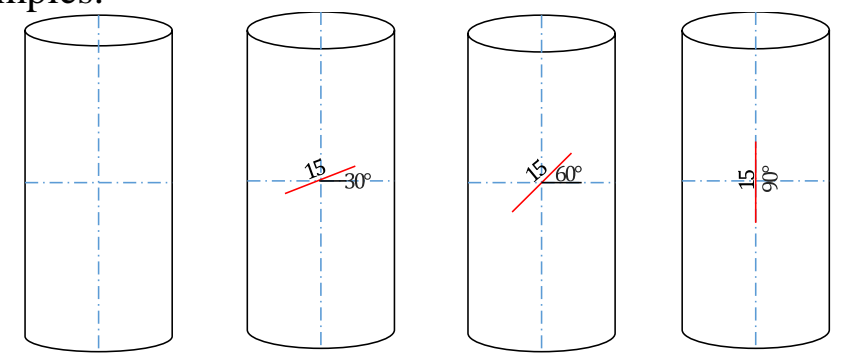

Fig. 1 Geometric parameters of the single fractured rock

F-T cycles were conducted with 0, 20, 40, 60 times, after that, quality and volume were measured, then uniaxial compression tests were carried out. The temperature range of F-T cycles was $-40^{\circ} \mathrm{C} \sim$ $+40^{\circ} \mathrm{C}$, each cycles of F-T was completed for $12 \mathrm{~h}(6 \mathrm{~h}$ for freezing and $6 \mathrm{~h}$ for melting). Freeze thaw cycle test equipment model type is KB-TH-S-150Z, uniaxial compression test equipment model type is RMT-201 rock and concrete mechanical testing machine made from Wuhan geotechnical engineering institute under Chinese Academy of Sciences. The experiments would be conducted by axial displacement control, and the displacement rate was $0.005 \mathrm{~mm} / \mathrm{s}$. The test results are given in Table 1.

Table 1 Compressive strength and elastic modulus under different freeze-thaw cycles

\begin{tabular}{|c|c|c|c|c|c|c|c|c}
\hline \multirow{2}{*}{ Group } & \multicolumn{4}{|c|}{ Uniaxial Compressive Strength /MPa } & \multicolumn{4}{c}{ Elastic Modulus /GPa } \\
\cline { 2 - 9 } & 0 & 20 & 40 & 60 & 0 & 20 & 40 & 60 \\
\hline W & 53.83 & 43.13 & 37.29 & 33.23 & 9.65 & 8.15 & 7.20 & 5.82 \\
\hline A1 & 42.15 & 40.26 & 34.96 & 17.24 & 4.90 & 3.62 & 2.58 & 2.29 \\
\hline A2 & 41.01 & 38.23 & 35.89 & 27.46 & 8.72 & 7.35 & 5.33 & 3.42 \\
\hline A3 & 54.59 & 49.86 & 48.04 & 33.09 & 9.01 & 6.17 & 5.35 & 4.27 \\
\hline
\end{tabular}

\section{Damage Evolution of Rock Masses under F-T Cycles and Load}

It's acknowledged, on basis of F-T experiments for fractured rock masses, that their mechanical property will decrease to different levels with increase of F-T cycles. On the other side, response of macro physical nature could reflect its internal damage in accordance with the theory of macro-phenomenological damage mechanics. Therefore, we choose elastic modulus as damage variable, which could reflect changing principle of rock mechanical and is easily to be measured in F-T experiments as well. Thus, variable of freeze-thaw rock damage is defined as follows:

$$
D_{n}=1-E_{n} / E_{0}
$$

Where, $E_{0}$ is the initial elastic modulus before F-T cycles, and $E_{n}$ is the one after the F-T cycles.

According to Lemaitre strain equivalent principle, Zhang ${ }^{[7]}$ brought out the extended equivalence principle, that is, damage condition from F-T is regarded as damage type 1, and damage condition from F-T and load as damage type 2. Then the constitutive relation between type 1 and type 2 is shown respectively as follows,

$$
\begin{aligned}
& \sigma_{n}=E_{0}\left(1-D_{n}\right) \varepsilon_{n} \\
& \sigma=E_{n}(1-D) \varepsilon
\end{aligned}
$$

Substituting Eq. (1) into Eq. (3), it obtains that:

$$
\begin{aligned}
\sigma & =E_{0}\left(1-D_{t}\right) \varepsilon \\
D_{t} & =D_{n}+D-D_{n} D
\end{aligned}
$$

Where, $D_{t}$ is combined damage from freezing, thawing and loading, $D_{n}$ is damage from both freezing and thawing while $D$ is damage only from loading, and $D_{n} D$ is the coupling. 
Taking damage from loading as a continuous process, and micro defaults inside rock masses is located randomly, when facing outside impacts, those micro defaults keep changing constantly and some of them will connect to each other. As a result, macro cracks happen and damage the rock. Supposed the rock micro-unit strength subject to Weibull distribution, the damage probability density function could be represented by material strain $\varepsilon^{[8]}$ as

$$
P_{(\varepsilon)}=\frac{m}{F}\left(\frac{\varepsilon}{F}\right)^{m-1} \exp \left[-\left(\frac{\varepsilon}{F}\right)^{m}\right]
$$

Where, $\varepsilon$ is the rock material strain, and $m, F$ are parameters of material physic mechanics. They are the same material mechanics parameters as elastic modulus $E$ and Poisson's ratio $v$.

Assuming, in any space $[\varepsilon, \varepsilon+d \varepsilon]$, the number of damaged micro-elements is $N P(x) \mathrm{d} x$. When the load increases to a level of $\varepsilon$, the number of damaged micro-elements $N_{s}$ can be obtained as ${ }^{[9]}$

$$
N_{s}=\int_{0}^{\varepsilon} N P(x) d x=N\left(1-e^{-\frac{1}{m}\left(\frac{\varepsilon}{\varepsilon_{m}}\right)^{m}}\right)
$$

It is assumed that the total number of internal micro-units in the rock micro- body is $N$, the number of damaged micro-units is $N_{s}$, and the statis- tical damage variable can be defined as

$$
D=N_{s} / N
$$

The damage variable $D$ can be calculated by substituting (7) into (8), it takes the following form.

$$
D=1-e^{-\frac{1}{m}\left(\frac{\varepsilon}{\varepsilon_{m}}\right)^{m}}
$$

Based on (1), (5) and (9), total damage can be determined by the following equation.

$$
D_{t}=1-\frac{E_{n}}{E_{0}} e^{-\frac{1}{m}\left(\frac{\varepsilon}{\varepsilon_{m}}\right)^{m}}
$$

In the above, $\varepsilon_{m}$ is strain parameter responding peak stress $\sigma_{m}$, and $m$ denotes the distribution concentration degree of strength in rock mass. $m=1 / \ln \left(E_{0} \varepsilon_{m} / \sigma_{m}\right)$.

It is acknowledged from Formula (10) that when we consider only F-T damage, rock load strain will be $\varepsilon=0, D_{m}=E_{n} / E_{0}=D_{n}$; when we only taking load damage into consideration, $E_{n}=E_{0}$,

$D_{m}=1-e^{-\frac{1}{m}\left(\frac{\varepsilon}{\varepsilon_{m}}\right)^{m}}=D$. Which are consistent with the previously mentioned.

\section{Calculated Examples and Model Validation}

Figure 2 states the evolving curves of damage mode for four different kinds of rocks under F-T and load, calculated through (10) and testing mechanics parameters and data. Table 2 shows $m$ and $\varepsilon m$ of the four various kinds of rocks under different number of F-T, and Figure 2 stands out the numbers of $E_{0}, E_{n}$ and $\sigma_{m}$.

Table 2 Damage evolution characteristic parameters of F-T samples

\begin{tabular}{ccccccccc}
\hline \multirow{2}{*}{ Group } & \multicolumn{9}{c}{$\varepsilon m / \%$ o } & \multicolumn{4}{c}{$\mathrm{m}$} \\
& 0 & 20 & 40 & 60 & 0 & 20 & 40 & 60 \\
\hline W & 7.85 & 6.89 & 7.12 & 7.56 & 2.93 & 2.31 & 1.64 & 1.27 \\
A1 & 11.78 & 11.17 & 9.86 & 8.78 & 3.18 & 3.26 & 3.09 & 1.09 \\
A2 & 6.35 & 6.50 & 6.79 & 7.18 & 3.32 & 2.54 & 2.00 & 1.21 \\
A3 & 8.44 & 7.45 & 7.86 & 8.04 & 3.20 & 3.36 & 2.58 & 1.28 \\
\hline
\end{tabular}




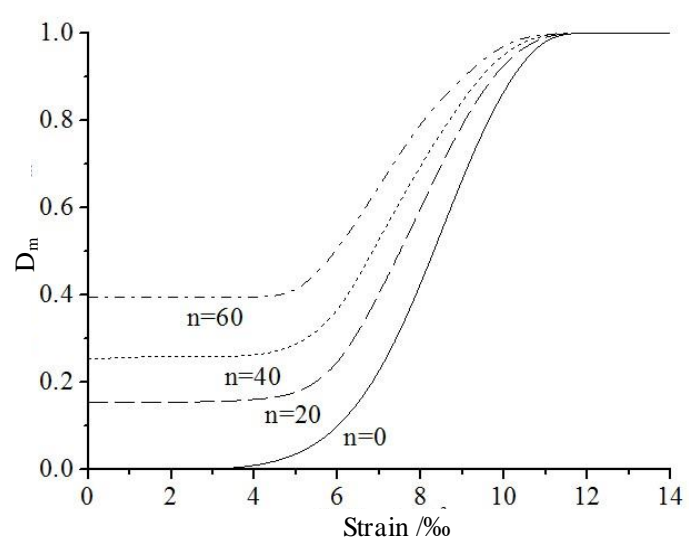

(a) W

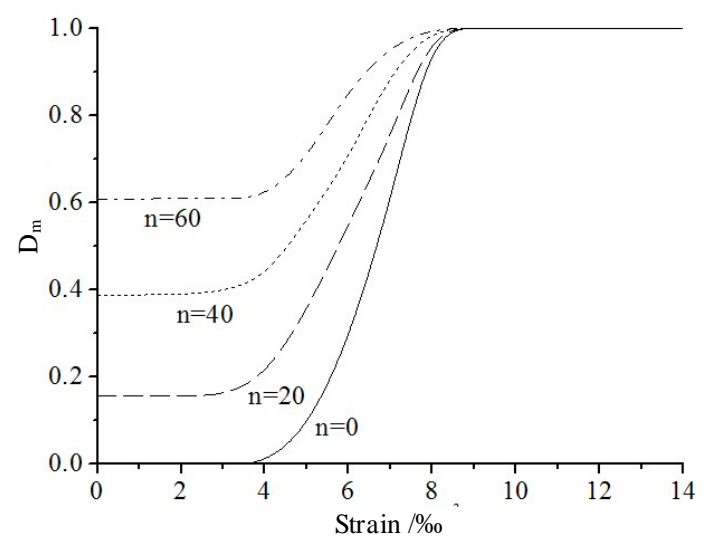

(c)A2

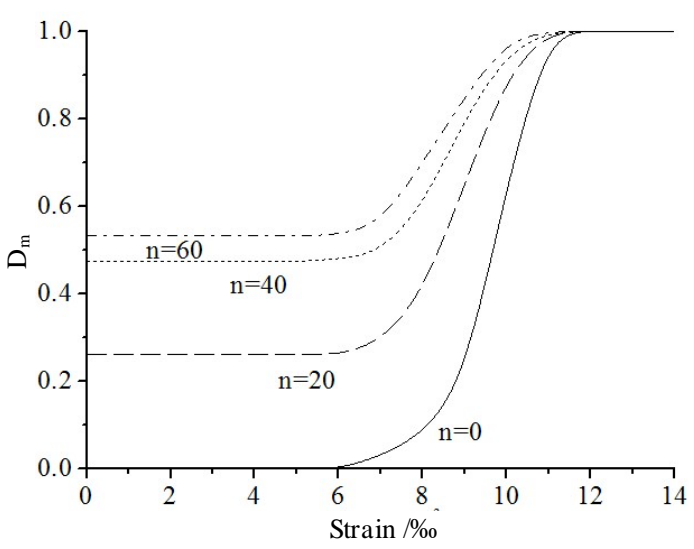

(b) A1

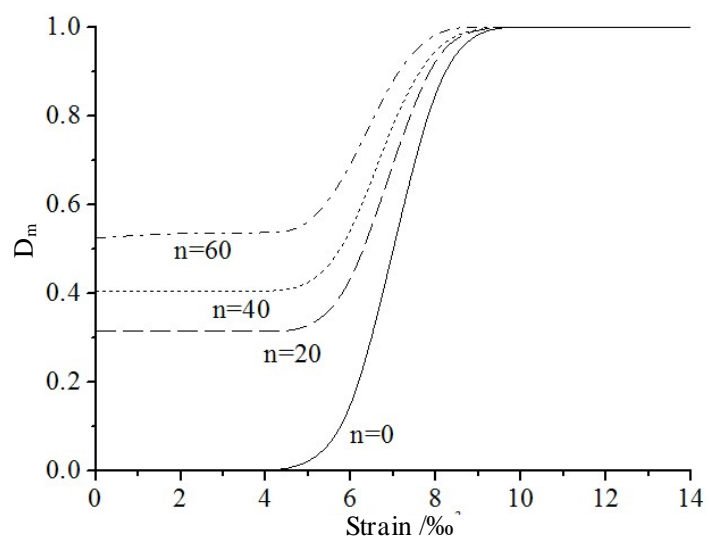

(d) A3

Fig.2 damage model evolution curves of cracked rocks under F-T and load condition It is found from figure 2 and table 2,

(1) The degree of damage for both intact and cracked rock samples intensify with the increase of F-T cycle. However, under the same times of F-T, intact sample appears not as damaged as cracked one, which means that existence of pre-cracks accelerate evolution of damage and initial damager has much effect on deterioration of damage of rock.

(2) The degree of damage for both intact and cracked rock samples intensify intensify with the increase of strain. At the initial period of being loaded, micro pores, micro cracks and pre-cracks are compressed as compact as the rate of cracks is quite small and intensity increases. With the loading stress become more and heavier, those micro cracks and pores develop gradually and damage worsens, till damage variable turns into 1 . As internal micro cracks appear, grow and then connect to each other, macro crack form, and strength of rock reaches its peak, then damage come out eventually.

(3) As freezing and thawing increase, compaction section extends accordingly. It is because the repeated freezing and thawing speed up the expansion of cracks and create new cracks at the same time, which make pores inside the rock become larger, micro crack connects to each other and then bigger cracks happen and damages come out eventually.

\section{Conclusions}

In this study, F-T test for 0, 20, 40 and 60 cycles were carried out on four different rock samples to evaluate their damage degree using a damage evolution model. Rock is assumed to be a damage material, and then we discuss the influence on the macroscopic and mesoscopic flaws on its mechanical behavior. Total damage is created by mixing two primary damage(F-T damage and load damage). The damage caused by the mesoscopic flaws is described by the damage evolution model based on Weibull distribution. Which can consider the effect of the mechanical property on the rock mass mechanical behaviors. 
On the basis of Lemaitre strain equivalence hypothesis, the coupled damage variable is obtained. Then the damage evolution model for rock mass under F-T and uniaxial compression tests is set up. Next, the proposed model is validated with the test data.

Test and analysis results indicate that the first 40 times freeze-thaw has little effect on uniaxial compressive strength, the strength significantly reduces after 60 times freeze-thaw, and then keeps stability later; crack dip angle has little effect on uniaxial compressive strength, other things being equal, the strength shows a trend of increase with the increase of crack dip angle.

Fractured rock suffers much less damage than intact rock with the stress level and the same times of freeze-thaw, damage degree of freeze-thaw rock will increase with the increase of strain and freeze-thaw cycles; Under the same condition of F-T cycles, the damage show little change with the increase of crack dip angle.

\section{Acknowledgements}

The research is financially supported by the National Natural Science Foundation of China (51274157), and Natural Science Foundation of Hubei Province (2014CFB575).

\section{References}

[1] Z. J. Shen. Weathering resistant design: An important aspect of future development of geotechnical engineering design[J]. Chinese Journal of Geotechnical Engineering, 2004, 26 (6) : 866-869.

[2] M. Mutlutürk, R. Altindag and G. Türk, A decay function model for the integrity loss of rock when subjected to recurrent cycles of freezing-thawing and heating-cooling. International Journal of Rock Mechanics and Mining Sciences. 2004, 41(2): 237-244.

[3] H. Yavuz, R. Altindag, S. Sarac, et al. Estimating the index properties of deteriorated carbonate rocks due to freeze-thaw and thermal shock weathering. International Journal of Rock Mechanics and Mining Sciences. 2006,43(5): 767-775.

[4] X.J. Tan, W.Z. Chen, H.M. Tian, et al. Laboratory investigations on the mechanical properties degradation of granite under freeze-thaw cycles. Cold Regions Science and Technology. 2011, 68(3): 130-138.

[5] G.S. Yang, D.Y. Xie, C.Q. Zhang, et al. CT analysis of mechanical characteristics of damage propagation of rock[J]. Chinese Journal of Rock Mechanics and Engineering, 1999,18(3): 250-254.

[6] J.Q. Mu, X.J. Pei, Y. Huang, et al. Experimental research on mechanical characteristics of rock with cycles of freeing-thawing action[J]. Journal of Engineering Geology, 2013, 21(01): $103-108$.

[7] Q.S. Zhang, G.S. Yang and J.X. Ren. New Study of Damage Variable and Constitutive Equation of Rock[J]. Chinese Journal of Rock Mechanics and Engineering, 2003, 2(1): 30-34.

[8] Weibull, W. 1951. A statistical distribution function of wide applicability. Journal of Applied Mechanics, 18: 293-297:

[9] W.G. Cao, Z.L. Fang, and X.J. Tang. A study of statistical constitutive model for soft and damage rocks. Chinese Journal of Rock Mechanics and Engineering, 1998, 17 (6): 628-633. 